This item was submitted to Loughborough's Research Repository by the author.

Items in Figshare are protected by copyright, with all rights reserved, unless otherwise indicated.

\title{
On the effects of directional bin size when simulating large offshore wind farms with CFD
}

\section{PLEASE CITE THE PUBLISHED VERSION}

http://dx.doi.org/10.1260/0309-524X.39.6.641

\section{PUBLISHER}

(C) Multi-Science Publishing

\section{VERSION}

AM (Accepted Manuscript)

\section{PUBLISHER STATEMENT}

This work is made available according to the conditions of the Creative Commons Attribution-NonCommercialNoDerivatives 4.0 International (CC BY-NC-ND 4.0) licence. Full details of this licence are available at: https://creativecommons.org/licenses/by-nc-nd/4.0/

\section{LICENCE}

CC BY-NC-ND 4.0

\section{REPOSITORY RECORD}

Argyle, Peter, and Simon J. Watson. 2019. "On the Effects of Directional Bin Size When Simulating Large Offshore Wind Farms with CFD". figshare. https://hdl.handle.net/2134/20573. 


\title{
On the Effects of Directional Bin Size when Simulating Large Offshore Wind Farms with CFD
}

\author{
Peter Argyle, Simon Watson \\ CREST, Loughborough University, ENGLAND \\ p.argyle@lboro.ac.uk
}

\begin{abstract}
Computational Fluid Dynamics (CFD) models are increasingly being used to simulate the flow through large wind farms to investigate scenarios with significant wake losses. This study investigates some of the effects caused by combining multiple simulation runs to represent the wide directional bin sizes sometimes required to ensure significant quantities of validation data and to account for wake meandering. CFD simulation results are combined using three different methods for six different directional bin sizes and compared against measurements from the Nysted offshore wind farm. Results show only small variation between averaging the model outputs uniformly or according to measured directional frequencies, whilst utilising a normal distribution reduces the variation between bin sizes. In addition, the greatest variation between results from combined multiple simulations compared to results from a single simulation occurs at the third turbine in a row. It is found that combined CFD results for the larger bin sizes are more accurate than the results for smaller bin sizes, when compared to the measured dataset with corresponding directional bin sizes.
\end{abstract}

\section{INTRODUCTION}

The significance of turbine wake effects on the available wind resource for large offshore wind farms has been well documented [1]. In order to accurately predict the production losses and increased fatigue caused by turbine wakes, a large number of computational techniques and models have been developed [2] [3]. As the more demanding simulation methods, such as the use of Computational Fluid Dynamics (CFD) models require large computational resources, it is often not cost effective to simulate every single combination of meteorological conditions. Therefore, relatively fast models such as WAsP [4] are used to assess the synoptic features whilst a CFD model is utilised to investigate key scenarios, such as wind flowing down a line of turbines. However, each method should be validated against field measurements, which can be expensive offshore and also time consuming to collect enough data from the less frequent wind speeds and directions. To reduce the time required for collecting field data, it is common practice to use directional data bins such as $x^{\circ} \pm y^{\circ}$, where $x$ is the wind direction of interest (such as the alignment of a row of turbines) and $y$ is the amount of deviation from $x$ which is deemed suitable for the analysis. When data are plentiful, $y$ is often small in order to reduce errors associated with horizontal wake expansion from other turbine rows, whilst $y$ is often increased to counter a lack of data. For example, datasets which require significant filtering to focus on specific parameters, such as stable atmospheric stability [5]. The use of directional bins are also used with steady state CFD simulations to help account for the effects of wake meandering.

As CFD model simulations can be time consuming, it is important to use best practice to minimise the number of runs required to accurately capture the observed farm wake losses. This work investigates the effects of varying the number and weighting of wind directions within combinations of CFD simulations for a line of turbines within a large offshore wind farm.

\section{METHOD}

For this work, results from the commercial CFD software package Ansys CFX Windmodeller [6] are analysed and compared with production data from the Nysted offshore wind farm in the Danish Baltic Sea. The wind farm consists of 72 turbines in a regular grid of 9 rows and 8 columns, with the columns offset such that the westerly 'down-the-line' direction investigated is $278^{\circ}$ as shown below 
in Figure 1. Production data are available from December 2006 through to February 2008 and are supplemented by meteorological data from Mast M1 for the same period. Although it is known that atmospheric stability significantly alters the turbine wake behaviour and is measureable using data from Mast M1 [7], there is no standardised method for categorising the results. Stability is also known to vary significantly within the lowest $100 \mathrm{~m}$ [8] and the required filtering process would significantly reduce the quantity of available data [9]. For this reason and for simplicity, this work neglects the effects of atmospheric stability.

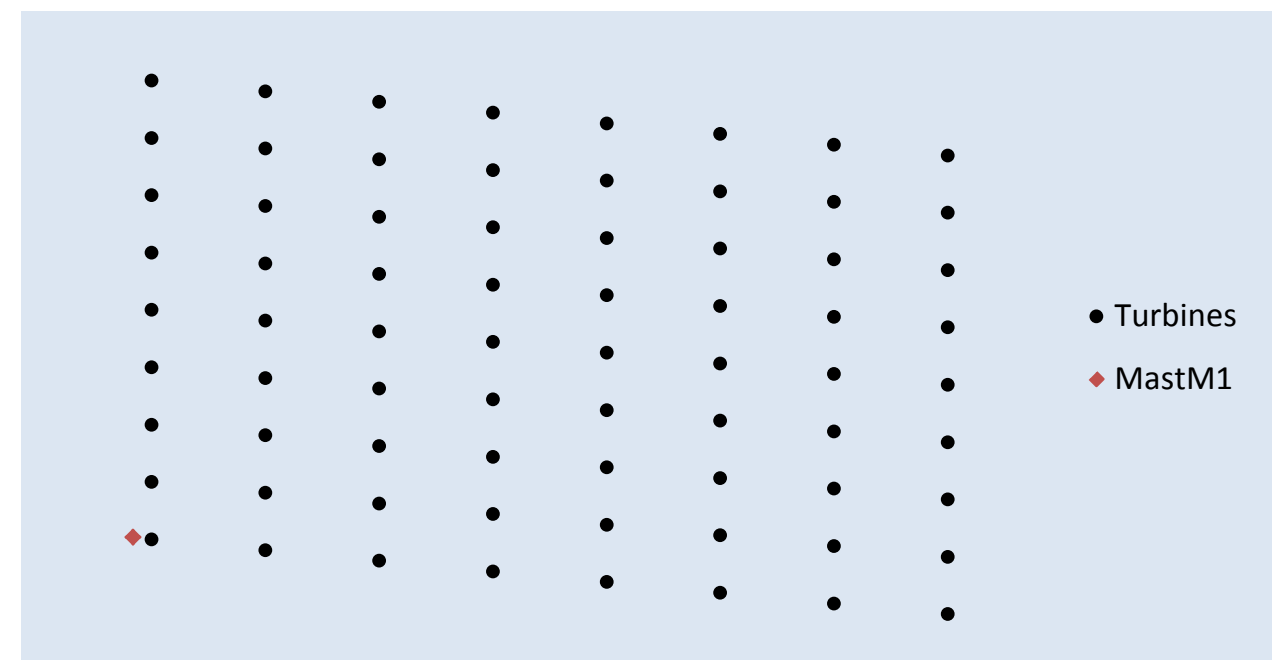

Figure 1 Layout of turbines and the meteorological mast M1 at the Nysted offshore wind farm. Turbine rows are separated by 5.9 rotor diameters (D) whilst the columns are separated by $10.4 \mathrm{D}$.

In order to increase the number of validation data events, each row of 8 turbines within the farm are considered as a subset, independent of the rest of the farm. Thus, if a turbine in the fourth row is undergoing maintenance, it is assumed this will not affect the productivity of turbines in other rows, although the whole fourth row of turbines are excluded from the analysis for the duration of the downtime. To prevent effects from the farm edges biasing the data, particularly for the larger directional bin sizes, both the most northerly and southerly rows of turbines are ignored. The production values for each 10-minute event are averaged together across the remaining 7 turbine rows (minus any rows with data problems during individual events). To help focus on wake effects and reduce variability in turbine wake strength, measured events were filtered to include only the events where the hub height wind speed measured at Mast M1 corresponds to high turbine thrust coefficient, at a wind speed of $7.5 \pm 1 \mathrm{~ms}^{-1}$. Despite the possible range of free-stream wind speeds considered for this study, the distribution of measured speeds was roughly uniform with a slight bias towards higher values giving an overall average speed of $7.7 \mathrm{~ms}^{-1}$.

The investigations into directional bin size will focus on the alignment of turbines in each row for Westerly flow, namely $278^{\circ}$. Six different case studies will be considered with a different bin size as shown in Table 1.

Table 1 List of direction bins used in this work

\begin{tabular}{|c|c|c|c|c|c|}
\hline Case Name & Directions Included & Bin Size & Events & Wind Speed & Colour \\
\hline Case A & $278 \pm 0.5^{\circ}$ & $1^{\circ}$ & 173 & $7.53 \mathrm{~ms}^{-1}$ & \\
\hline Case B & $278 \pm 1.5^{\circ}$ & $3^{\circ}$ & 485 & $7.60 \mathrm{~ms}^{-1}$ & \\
\hline Case C & $278 \pm 2.5^{\circ}$ & $5^{\circ}$ & 687 & $7.64 \mathrm{~ms}^{-1}$ & \\
\hline Case D & $278 \pm 3.5^{\circ}$ & $7^{\circ}$ & 977 & $7.67 \mathrm{~ms}^{-1}$ & \\
\hline Case E & $278 \pm 4.5^{\circ}$ & $9^{\circ}$ & 1238 & $7.68 \mathrm{~ms}^{-1}$ & \\
\hline Case F & $278 \pm 5.5^{\circ}$ & $11^{\circ}$ & 1368 & $7.68 \mathrm{~ms}^{-1}$ & \\
\hline
\end{tabular}


Many assumptions are often made about the offshore wind climate to help simplify models or data analysis. An example of this is the assumption of measurement data to be either uniformly or normally distributed, such as displayed in Figure 2, where the normal distribution has three standard deviations above and below the mean value within the bin size defined by Table 1.
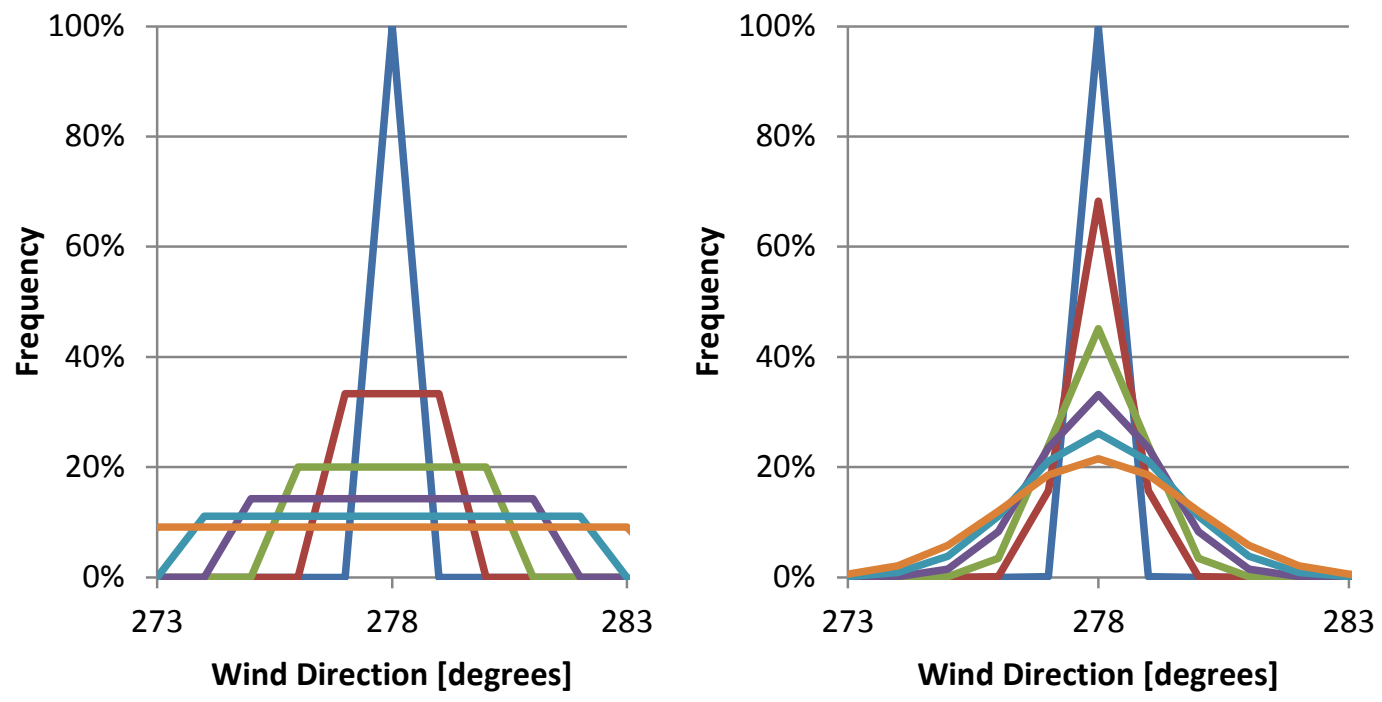

$\longrightarrow$ Case A Case B Case C Case D Case E Case F

Figure 2 Expected directional frequencies for each case where directions are uniformly distributed (Left) or normally distributed (Right)

In reality, the measured frequencies for this directional sector at the Nysted farm (shown in Figure 3) are closer to the uniform distribution, although some deviation does exist.

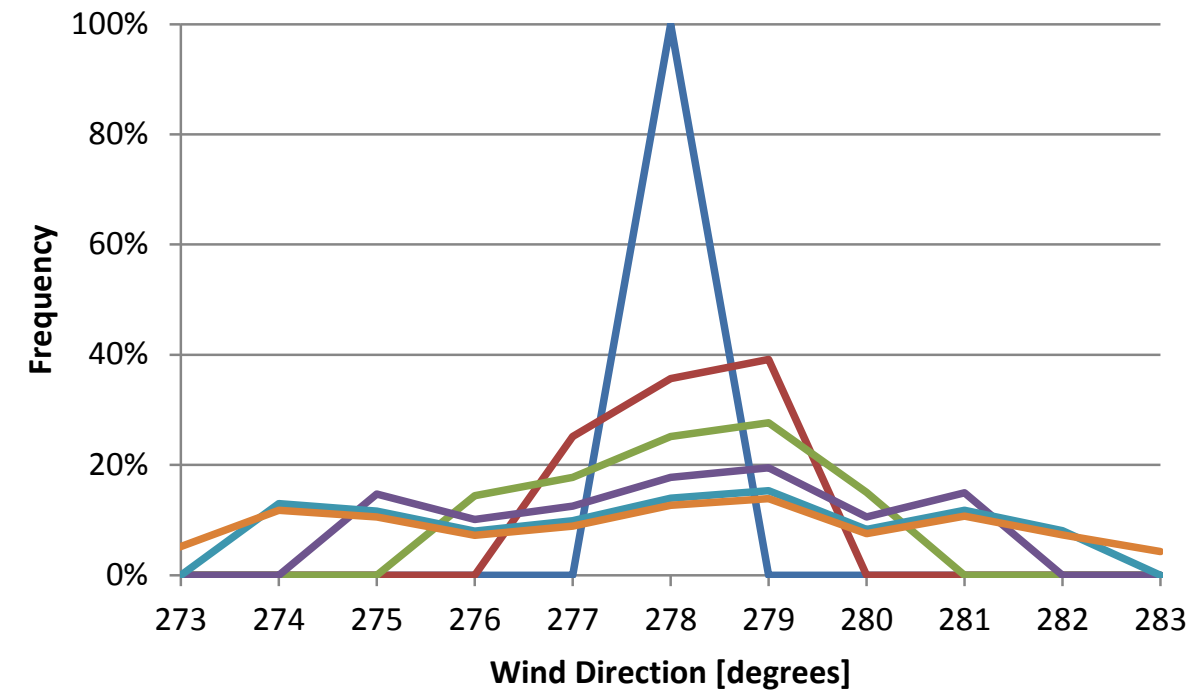

Case A Case B Case C Case D Case E Case F

Figure 3 Measured directional frequencies for each case

The frequency distributions above suggest that for larger bin sizes, events aligned directly down the row of turbines occur less frequently than events where the flow alignment is offset by a few degrees. This implies that average wake losses for the larger bin sizes such as Case F should be significantly lower than the average wake losses for the narrow bin sizes where the turbines are always exposed to the centre of the wake and thus the highest levels of turbulence and lowest flow 
speeds. However, Figure 4 below shows there is not much difference between the normalised power ratios through the farm for each bin size and therefore suggests the variation in wind directions within even a $11^{\circ}$ bin size may not be significant. However, when the power generation for each bin size is compared against that of Case A rather than against the free-stream generation value, significant variation in the measurements is observed. This is partially the result of changing sample sizes and partially due to varying the significance of turbine wakes in overall production.
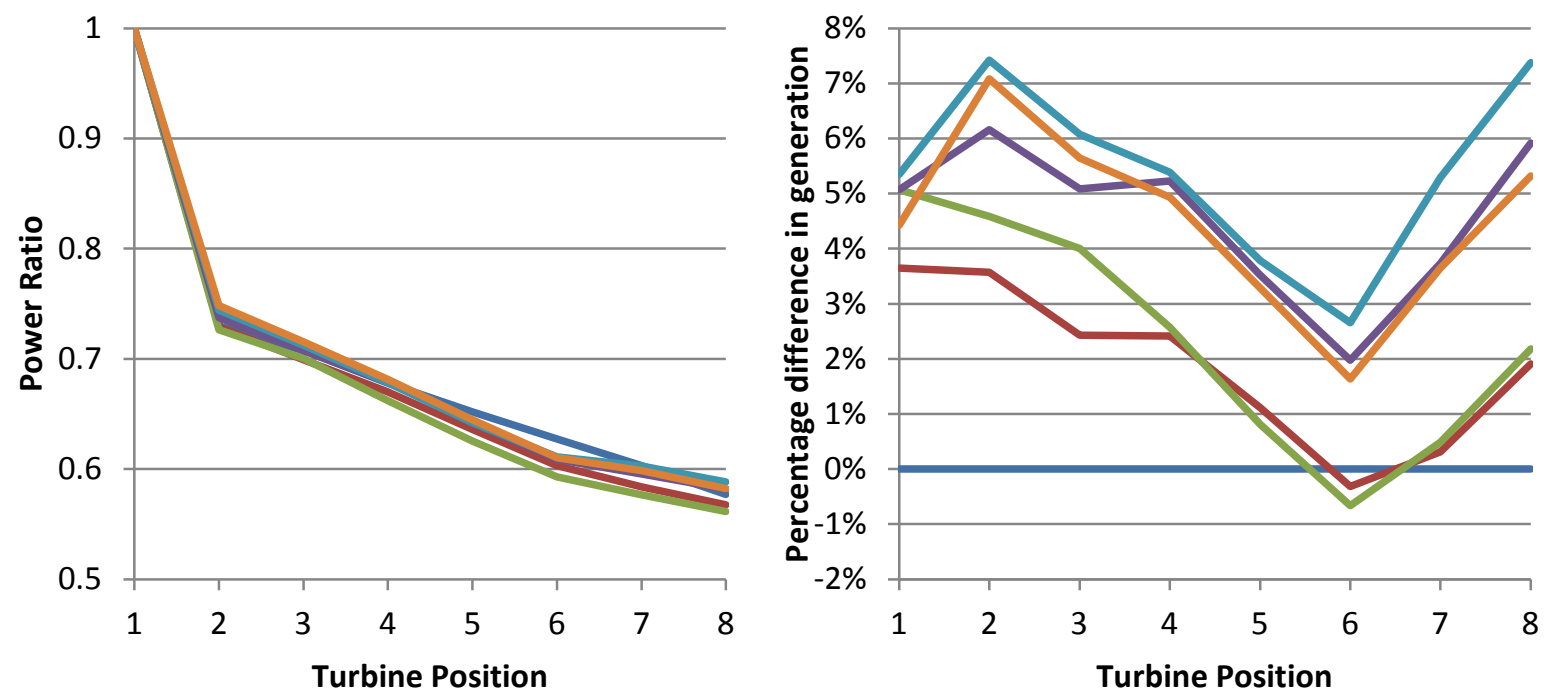

Case A Case B Case C Case D Case E Case F

Figure 4 Left: Average measured power generated by each turbine position, normalised by the production of the free-stream turbine. Right: Percentage difference between each Case and Case A

Three different turbulence models were considered in this work. The first two (hereafter referred to as Model_1 and Model_2) are $k$ - $\omega$ shear stress transport (SST) turbulence models [10] using a two equation eddy-viscosity approach for incompressible/compressible turbulent flow, each using a different set of values for key constants as shown below in Table 2 where the subscripts 1 and 2 indicate the $\omega$ and $k$ model regimes respectively. The third model by comparison (hereafter referred to as Model_3) uses a $k-\varepsilon$ turbulence model with an Unsteady Reynolds-Averaged Navier-Stokes (URANS) approach [11] including the Coriolis force as a difference to the geostrophic balance in the momentum equations. Although Model_3 is capable of incorporating the effects of buoyancy as a function of potential temperature, neutral stability conditions are used for consistency with the measurements and other models.

Table 2 The set of constants used in each SST simulation

\begin{tabular}{|l|c|l|l|c|c|c|c|c|c|c|c|}
\hline & $C_{\mu}$ & $t d_{\exp 1}$ & $t d_{\exp 2}$ & $C_{\varepsilon 1}$ & $C_{\varepsilon 2}$ & $\sigma_{k 1}$ & $\sigma_{k 2}$ & $\sigma_{\omega 1}$ & $\sigma_{\omega 2}$ & $\beta_{1}$ & $\beta_{2}$ \\
\hline Model_1 & 0.09 & 1.2 & 1.087 & 1.44 & 1.92 & 1.176 & 1 & 2.0 & 1.167 & 0.075 & 0.0828 \\
\hline Model_2 & 0.03 & 0.6 & 0.6 & 1.44 & 2.66 & 1.176 & 1 & 0.871 & 0.795 & 0.05 & 0.05 \\
\hline
\end{tabular}

\section{RESULTS}

The simulation results from Model_1, Model_2 and Model_3 are shown in Figure 5, Figure 7 and Figure 8 respectively. Comparing the modelled power ratios against the measured values displayed in Figure 4, reveals that both the SST models significantly overestimate the turbine wake effects whilst Model_3 slightly underestimates them. This is consistent with results found by [5]. However the power ratio graphs in Figure 5 do show that simulated wind farm productivity does vary with both directional bin size and the averaging method. Typically, the wider bin sizes lead to higher power ratios throughout the farm, but most noticeably between turbine positions 2 and 5 . The onset of the region where the rate of wind energy extracted from the hub height flow is balanced by that 
drawn down to replace it from above, is also visibly delayed further into the farm. For example, in each of the three left hand graphs in Figure 5 the power ratio for Case $A$ reaches a minimum value at roughly turbine position 5 whilst the value for Case $F$ is still decreasing at turbine position 8 . Figure 5 confirms that the method used to combine the output from multiple CFD simulations with different flow directions, is a significant factor in the overall resulting power ratio values. For example, combining Model_1 results from each direction in uniform manner for Case $\mathrm{F}$ at turbine position 3 returns a power ratio of 0.57 whilst combining the exact same simulation output using a normal distribution returns a power ratio of 0.50 , a $12 \%$ difference. The significance of method used to combine results decreases with smaller bin sizes; using the same example as before but for Case $C$, the difference in power ratio is only $4 \%$. By weighting each of the model outputs according to the directional frequencies measured on site, the resulting power ratios are different again, although they tend towards the uniformly weighted values, especially for the larger bin sizes.

\section{Model_1}
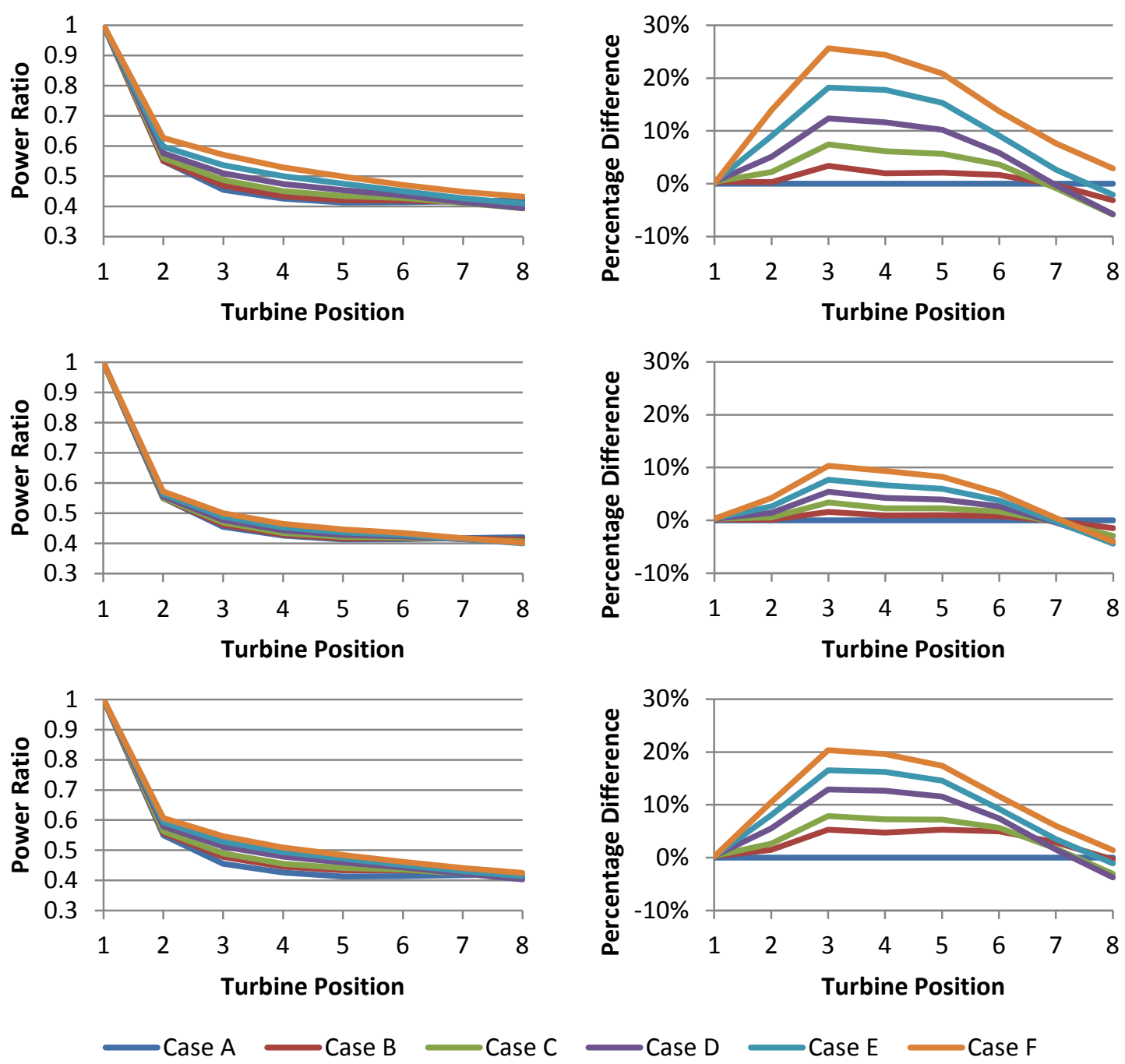

Figure 5 Results from Model_1. Each wind direction has been given a weighted influence according to Top: A uniform distribution, Middle: A normal distribution, Bottom: The measured frequencies in Figure 3. Left: Normalised simulated power production at each turbine position, Right: Percentage difference between turbine production results for each simulated Case compared to Case $A$.

Unlike the measured percentage differences in Figure 4, each of the graphs displaying simulated results above show zero variation in the free-stream turbine output with varying directional bin sizes. This is to be expected as there is no variation in simulated hub height wind speed upstream of the 
first actuator disks used to represent turbines; therefore the small variations in wind direction has no impact on results. This contrasts with the variation in measured production values from the Nysted farm when directional bin sizes are changed. As the dataset was filtered to restrict wind speed values to the range $7.5 \pm 1 \mathrm{~ms}^{-1}$, it is possible that some events included in the wider bin sizes were consistently higher than for the central bin, Case A. This theory is supported by Table 1 which shows the average wind speed increasing with bin size. The largest percentage difference between Cases always occurs at turbine position 3 , the furthest turbine into the farm which experiences the free-stream flow for at least some of its rotor at wide flow angles. Turbines further into the farm are affected by expanding wakes from neighbouring turbine rows which block the faster moving flow. This theory is supported by Figure 6 which visualises the hub height wind speed values for Model_1 flow angles $278^{\circ}$ and $283^{\circ}$. Note that the fourth turbine also experiences some fast moving flow, though the flow is partially affected by the freestream turbine in the neighbouring row, and so produces significantly more power than the same turbine for flow direction $278^{\circ}$, but less than the turbine at position three. It is also worth noting that Figure 5 shows that averaging simulation results across mid-size bin sectors sometimes leads to lower values than just using the value from Case $A$ at turbine position eight. However the wider directions in Cases $\mathrm{E}$ and $\mathrm{F}$ balance this effect and as there are no data after turbine position eight, it is not possible to show whether this effect would continue in a larger farm. In contrast to results from the simulations, measured values shown in Figure 4 indicate the peak in percentage difference between Case A and wider bin sizes occurs at turbine position two rather than three, and that the percentage differences significantly increase again after a minimum at position six.

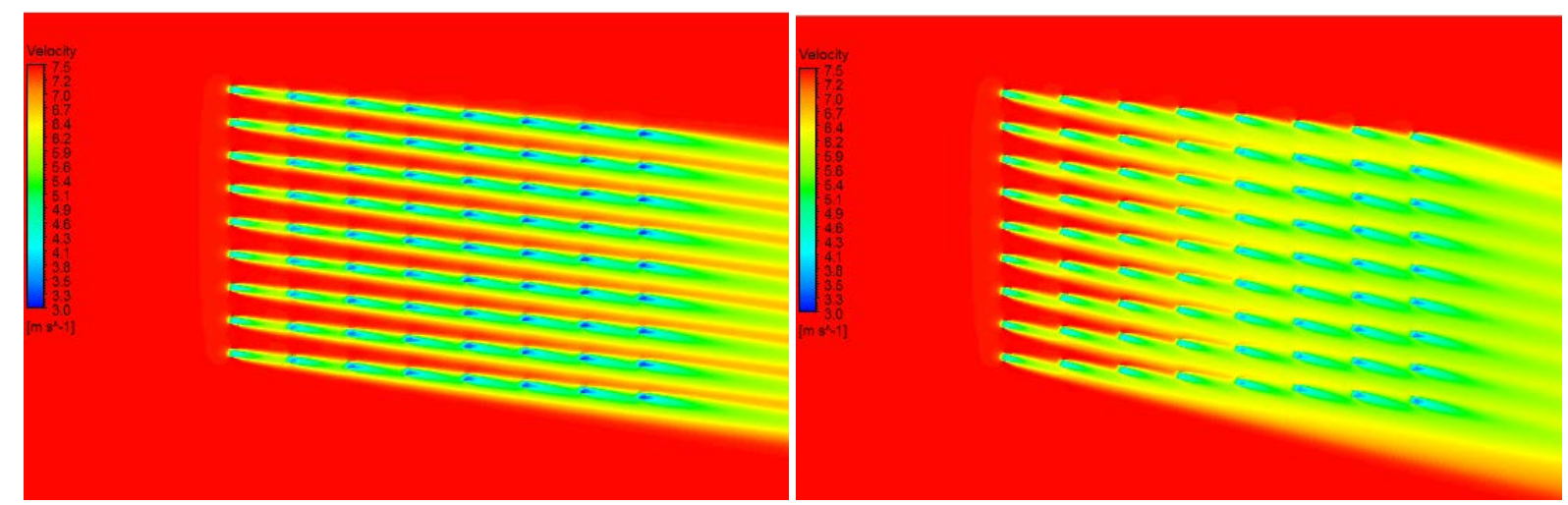

Figure 6 Wind speed results from Model_1 for wind directions $278^{\circ}$ (Left) and $283^{\circ}$ (Right)

Results from Model_2 are shown in Figure 7. As the SST model used is very similar to Model_1, the power ratio graphs are very similar, though with a subtle increase in wake losses for the wider directional bins in the front half of the farm, whilst subtle decreases in wake losses exist across all bin sizes by turbine seven.. The three different methods of combining simulation results across the bin directions results in the same pattern as for Model_1, the uniform distribution method leads to greatest variation in power ratios across bin sizes whilst the normal distribution leads to the smallest variation in power ratios. The graphs showing percentage differences for Model_2 are also very similar to those for Model_1, indicating the most significant variation from Case A happens for uniformly weighted Case $\mathrm{F}$ results whilst the normally distributed results again show least deviation from Case A. However, the values for percentage difference at turbine position four with Model_2 are higher than their counterparts for Model_1, suggesting the change in turbulence model constants may subtly decrease the predicted wake losses once the wakes have merged horizontally. 

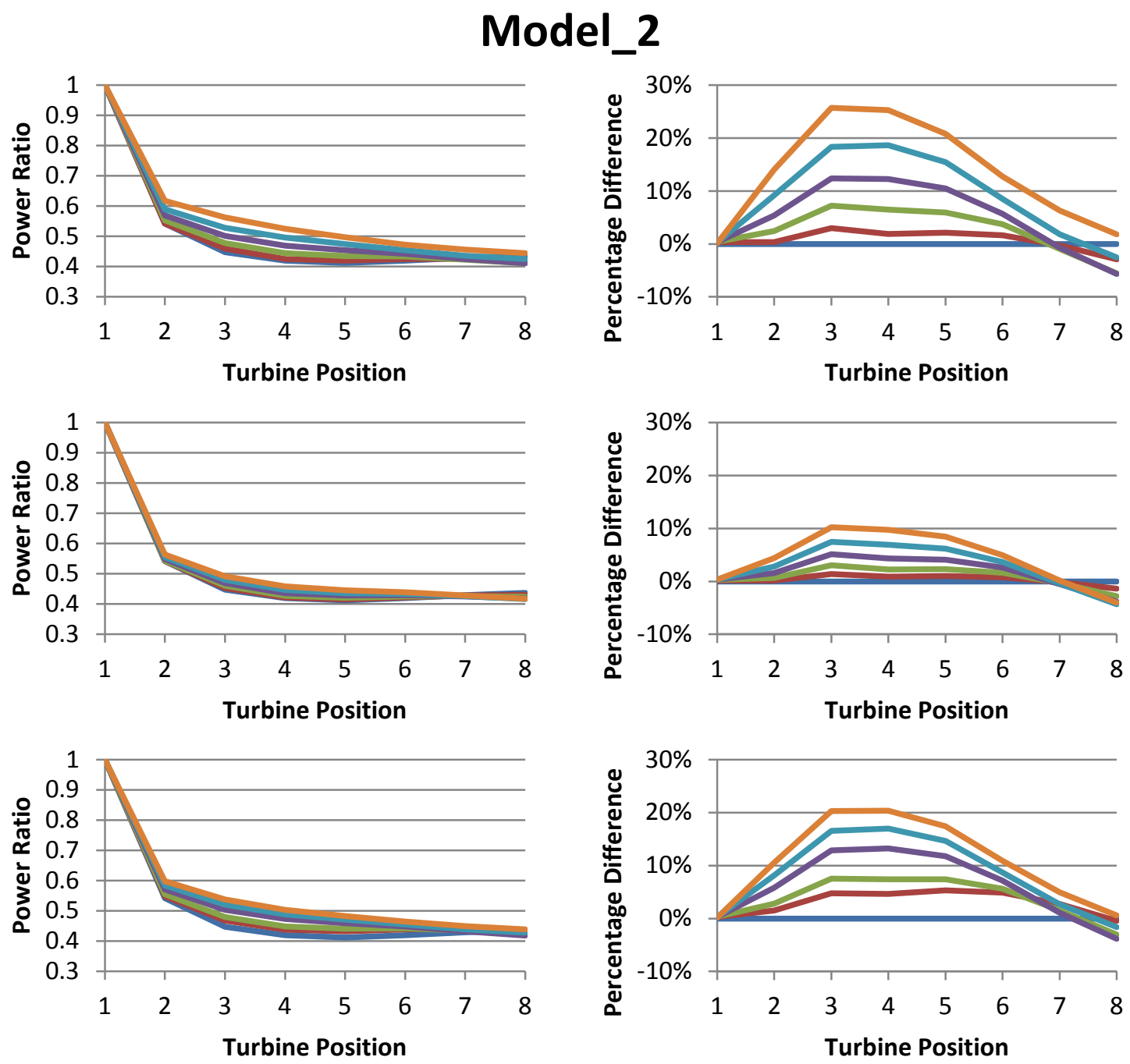

Case A Case B Case C Case D Case E Case F

Figure 7 Results from Model_2. Each wind direction has been given a weighted influence according to Top: A uniform distribution, Middle: A normal distribution, Bottom: The measured frequencies in Figure 3. Left: Normalised simulated power production at each turbine position, Right: Percentage difference between turbine production results for each simulated Case compared to Case $A$.

Results from Model_3 are shown in Figure 8. Note that the scale on each vertical axis is different to those in Figure 5 and Figure 7 in order to highlight the significant decrease in variation between averaging techniques in comparison to the other models. Once again the option of averaging the individual simulation results in a uniform manner results in the largest variation with bin size, though significantly less than for either Model_1 or Model_2. Similarly, weighting the directional results to fit a normal distribution again results in the least variation with bin size, whilst weighting the simulations by the measured frequencies leads to results similar to the uniformly weighted option. The Model_3 results differ significantly from those for the SST options relating to how the amount of variation from Case A changes with distance into the farm. For example, results from Model_1 and Model_2 all display a maximum percentage difference from Case A at turbine position three before reducing with distance into the farm with values close to $0 \%$ at either position seven or eight. By contrast, whilst the results from Model_3 also show a maximum positive variation from Case A at turbine position three, there is also a maximum negative variation at position seven for each bin size of similar magnitude. This suggests significance of bin size, and thus to a lesser extent the averaging technique, may varies in a sinusoidal pattern with depth through the farm, although this is likely to be strongly dependent on farm layout. 


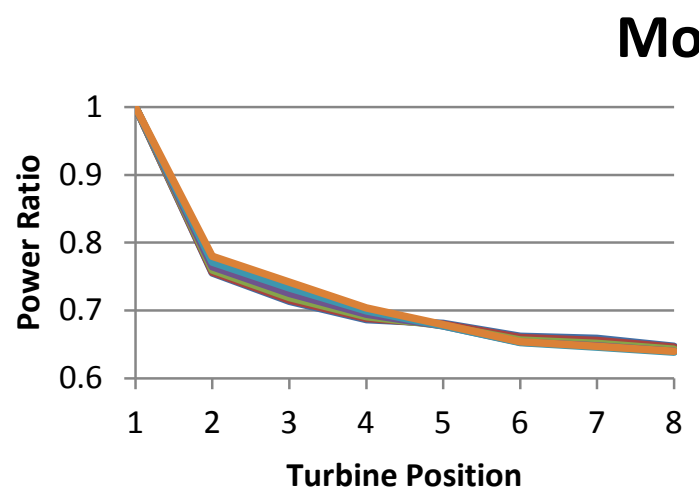

Model_3
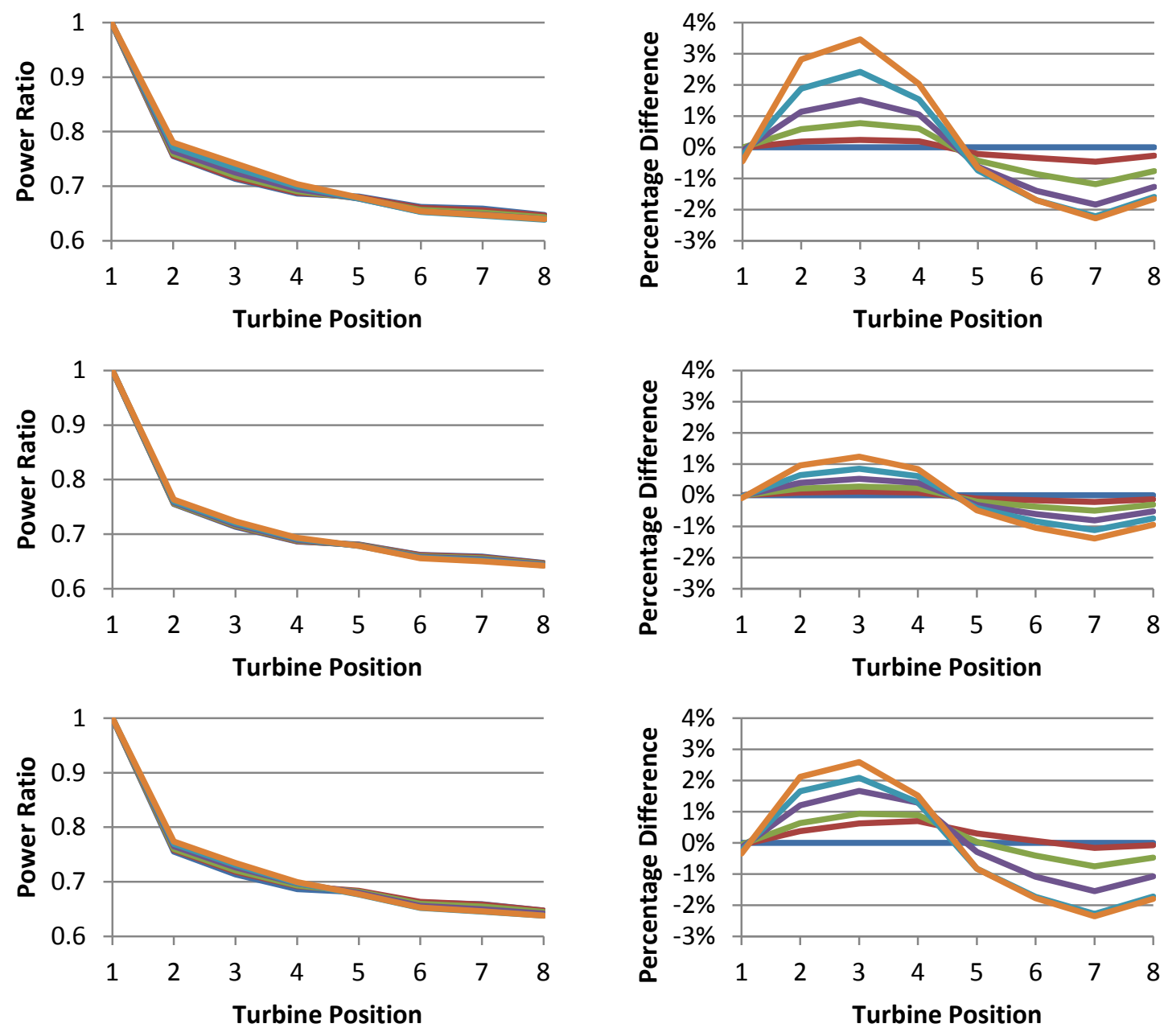

Case A Case B Case C Case D Case E Case F

Figure 8 Results from Model_3. Each wind direction has been given a weighted influence according to Top: A uniform distribution, Middle: A normal distribution, Bottom: The measured frequencies in Figure 3. Left: Normalised simulated power production at each turbine position, Right: Percentage difference between turbine production results for each simulated Case compared to Case $A$.

Finally, the output from Model_3 averaged via each method, are compared below as a percentage difference from the values measured at the Nysted farm for the corresponding bin sizes. Note that there is a small difference in results from the normal distribution method for Case A in Figure 9 as the distribution was consistently defined to ensure the bin represented $95 \%$ of the data, corresponding to the mean direction $\left(278^{\circ}\right)$ and three standard deviations each side. This aspect of the normal distribution may also account for some of the slight difference in values below for Case $B$, however to a lesser extent as indicated by Figure 2. None of the graphs show a percentage difference of zero for turbine position one since the accepted wind speed bin for measured data was $2 \mathrm{~ms}^{-1}$, variation not accounted for by the simulations. Whilst the approach using the normal distribution always returns the smallest values for turbine positions two to four, it is rarely the most accurate method for bin sizes greater than $3^{\circ}$. However, as there is negligible difference in results between the uniformly distributed and measurement weighted methods until the bin sizes exceed $7^{\circ}$, Figure 9 suggests there is little to be gained from combining CFD results from multiple directions in a non-uniform manner for this particular case study. 


\section{Difference between Model_3 Simulation and the Measured Values}
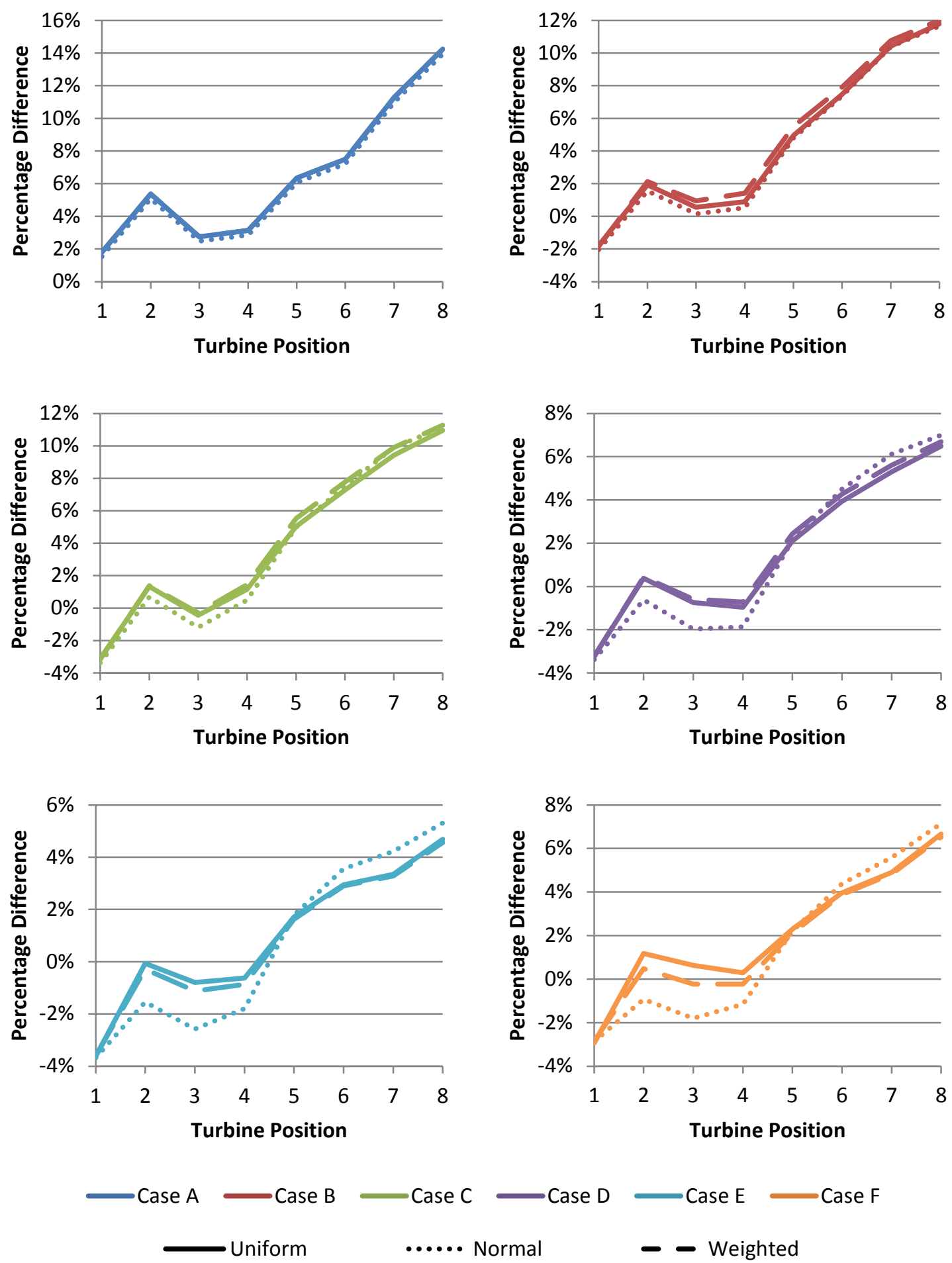

Figure 9 Plots showing the percentage difference between the averaged simulated power generation using Model_3 results via the three separate methods, and the measured values from the Nysted wind farm. Each graph corresponds to a different bin size as indicated by its colour, whilst the solid, dotted and dashed lines indicate results using the uniform, normal and measurement weighted distributions. 


\section{CONCLUSION}

This work has compared how the utilisation of three separate methods for combining results from multiple CFD simulation flow directions, can affect the overall analysis of turbine wake losses within a large offshore wind farm. The results from three different turbulence models all showed that averaging the individual simulation results uniformly across each direction within the relevant bin size, lead to the highest rates of variation with changing bin size. Similarly, all three models showed that by averaging the individual simulation results according to the normal distribution, the lowest rates of variation with changing bin size were achieved. Weighting the CFD results according to their measured directional frequencies at the Nysted farm resulted in simulated wake losses similar to the uniformly distributed results. However, the similarity between these two options is likely to be dependent on both the site and period of data used to determine directional frequencies. The peak in percentage difference between wind flow aligned with the turbine rows and results from larger bin sizes, which occurs at turbine position three is also likely to be site dependent as it is linked to the turbine separation. However, it is reasonable to assume that any array of turbines with a regular layout will display a similar feature whilst the sinusoidal appearance of the percentage difference with distance through the farm with Model_3 suggests any variation in results caused by bin size selection may cancel themselves out for a large enough farm. Finally, it was shown that when considering the farm as a whole rather than on an individual turbine basis, using larger directional bin sizes for sampling the raw data and proportionally more CFD simulations leads to a significantly more accurate representation of wind flow through a farm. However, utilising larger directional bin sizes comes at the cost of greater computational resources required to generate the additional CFD results, though this may be compensated for by less time required for the collection of a suitably sized field dataset.

As this work was been based on data from only one wind farm, it is probable that the significance of these results will vary with farm layout design. They will also likely vary for higher wind speeds where the turbine wake effect is smaller. It is recommended that a comparative study be conducted for an alternative wind farm, with a focus on investigating possible variation caused by the effects of atmospheric stability which is known to be a significant factor for understanding turbine wake development and subsequent losses in offshore wind farms.

\section{AKNOLEDGMENTS}

The Authors would like to thank E.ON for sponsoring this research through an EPSRC CASE award and the EPSRC Supergen Wind Energy Technologies consortium (grant number: EP/H018662/1).

\section{REFERENCES}

[1] L. Jensen, C. Mørch, P. Sørensen and K. Svendsen, "Wake Measurements from the Horns Rev Wind Farm," in EWEA Conference and Exhibition, London, 2004.

[2] B. Sanderse, S. van der Pijl and B. Koren, "Review of Computational Fluid Dynamics for Wind Turbine Wake Aerodynamics," Wind Energy, vol. 14, pp. 799-819, 2011.

[3] R. Stevens, D. Gayme and C. Meneveau, "Coupled Wake Boundary Layer Model of Wind-Farms," Journal of Renewable and Sustainable Energy, vol. 7, 2015.

[4] R. Barthelmie, L. Folkerts, G. Larsen, K. Rados, S. Pryor, S. Frandsen, B. Lange and G. Schepers, "Comparison of Wake Model Simulations with Offshore Wind Turbine Wake Profiles Measured by Sodar," Journal of Atmospheric Oceanic Technology, vol. 23, pp. 888-901, 2006.

[5] P. Argyle, PhD Thesis: Computational Fluid Dynamics Modelling of Wind Turbine Wake Losses in Large Offshore Wind Farm, Incorperating Atmospheric Stability, Loughborough University, 2015.

[6] C. Montavon, I. Jones, C. Staples, C. Strachan and I. Gutierrez, "Practical Issues in the use of CFD for Modelling Wind Farms," in EWEA Conference and Exhibition, Marseille, 2009.

[7] R. Barthelmie, S. Frandsen, P. Rethore and L. Jensen, "Analysis of Atmospheric Impacts on the 
Development of Wind Turbine Wakes at the Nysted Wind Farm," in EWEA Offshore Conference and Exhibition, Berlin, 2007.

[8] P. Argyle and S. Watson, "Assessing the Dependence of Surface Layer Atmospheric Stability on Measurement Height at Offshore Locations," Journal of Wind Engineering and Industrial Aerodynamics, vol. 131, pp. 88-99, 2014.

[9] P. Argyle and S. Watson, "A Study of the Surface Layer Atmospheric Stability at Two UK Offshore Sites," in EWEA Conference and Exhibition, Copenhagen, 2012.

[10] F. Menter, "Two-Equation Eddy-Viscosity Turbulence Models for Engineering Applications," AIAA Journal, vol. 32, no. 8, pp. 1598-1605, 1994.

[11] C. Montavon, I. Jones and C. Sander, "Accounting for Stability Effects in the Simulation of Large Array Losses," in DEWEK, Bremen, 2012.

[12] Y. Wu and P. Fernando, "Simulation of Turbulent Flow Inside and Above Wind Farms: Model Validation and Layout Effects," Boundary Layer Meteorology, vol. 146, pp. 181-205, 2013. 